\title{
Isotope-resolved photodissociation pathways of lead-doped bismuth clusters from tandem multi-reflection time-of-flight mass spectrometry
}

\author{
Paul Fischer* and Lutz Schweikhard \\ Institut für Physik, Universität Greifswald, 17487 Greifswald, Germany
}

(Received 29 May 2019; published 28 October 2019)

\begin{abstract}
The photofragmentation behavior of the lead-doped bismuth cluster ${ }^{209} \mathrm{Bi}_{7}{ }^{208} \mathrm{~Pb}^{+}$is investigated by use of multi-reflection time-of-flight mass spectrometry (MR-ToF MS), which serves both as precursor selector on a scale of single atomic mass units as well as high-resolution fragment analyzer. This is an implementation of a tandem MR-ToF study, where two instances of high-resolution mass spectrometry are used, namely for pre-selection and post-excitation analysis, in quick succession within a single device to determine complex dissociation patterns. The full information of the fragmentation pathways is obtained, including branching ratios describing the retention of the charge on the pure or compound cluster. The results are compared to the behavior of the analogous pure bismuth cluster ${ }^{209} \mathrm{Bi}_{8}^{+}$and interpreted within the framework of well-established cluster stability rules.
\end{abstract}

DOI: 10.1103/PhysRevResearch.1.033050

\section{INTRODUCTION}

The properties of atomic clusters [1,2], systems located between the single atom and bulk matter, vary with their number of atoms and valence electrons. For monoatomic species, these values are inherently coupled unless different charge states are addressed. Binary systems, i.e., with atoms of two different elements, offer more freedom, as the number of atoms of the second species becomes an additional tuning parameter. Alkali-metal clusters doped with other alkali $[3,4]$ or metal species [3,5-7] were already studied in the 1980s and 1990s. Since then, research of bimetallic clusters has been expanded significantly [8-11], with topics of interest being as diverse as, e.g., the fine-tuning of catalytic processes [12-15] and even biomedical markers [16].

Many interaction-based studies call for two instances of mass-spectrometric analysis, commonly referred to as tandem mass spectrometry, MS/MS or $\mathrm{MS}^{2}$ [17]: once prior to the interaction to select the precursor and again afterward to resolve and analyze the reaction products. Bimetallic systems are experimentally particularly challenging, as the mass resolving power needed for compound-cluster investigations often surpasses that required for single-element species by orders of magnitude. To increase the resolving power of timeof-flight (ToF) devices [18], the principle of multi-reflection (MR) has been introduced [19] akin to the concept of electrostatic ion beam traps (EIBTs) consisting of two opposing ion mirrors [20,21]. In nuclear physics, MR-ToF devices have recently been applied for fast mass separation or ion selection

\footnotetext{
*Corresponding author: paul.fischer@uni-greifswald.de

Published by the American Physical Society under the terms of the Creative Commons Attribution 4.0 International license. Further distribution of this work must maintain attribution to the author(s) and the published article's title, journal citation, and DOI.
}

[22,23] as well as precision mass measurements [24]. On the molecular level, they are appreciated for their capability as ion traps with a virtually unrestricted mass range, operated either in "non-bunching mode," where injected ion beams fill the entire trap volume [25,26], or with capacitive pickup detection (Fourier transform MR-ToF) for high-resolution mass spectrometry [27-30].

Here, we introduce the dual use of high-resolution multireflection time-of-flight mass separation and spectrometry in the same measurement cycle of a single device. This method constitutes a substantial extension of a recently reported approach to MR-ToF- or EIBT-based photofragmentation studies [31], which is briefly reviewed as the present method is based upon it.

\section{EXPERIMENTAL SETUP}

Metal clusters are produced via laser ablation [32-35], accelerated to $2 \mathrm{keV}$, and captured between two electrostatic ion mirrors with an in-trap lift electrode [36] (see Fig. 1 for experimental setup). In previous work [31], precursor bismuth cluster ions are selected on a scale of single atomic mass units employing the MR-ToF device's high resolving power. This is necessary in order to remove all other "contaminants," in particular those where a bismuth atom (mass number $A=$ $209)$ is replaced by a lead atom $(A=204,206,207,208)$ due to impurities of the ablation target (see Fig. 10 in [37]). After 400 revolution periods (some tens of milliseconds depending on cluster size), the isobar-selected precursor is irradiated by a laser pulse. The pulse timing is synchronized such that the ion bunch is located at its turnaround point in the trap's entry-side mirror potential, implying a kinetic energy of (close to) zero at the time of laser interaction. Fragment ions thus have the same energy as the precursor regardless of mass and can be studied with the same post-trap detector configuration.

The investigations reported in Ref. [31] are limited to clusters consisting only of ${ }^{209} \mathrm{Bi}$ atoms, as bismuth has just 


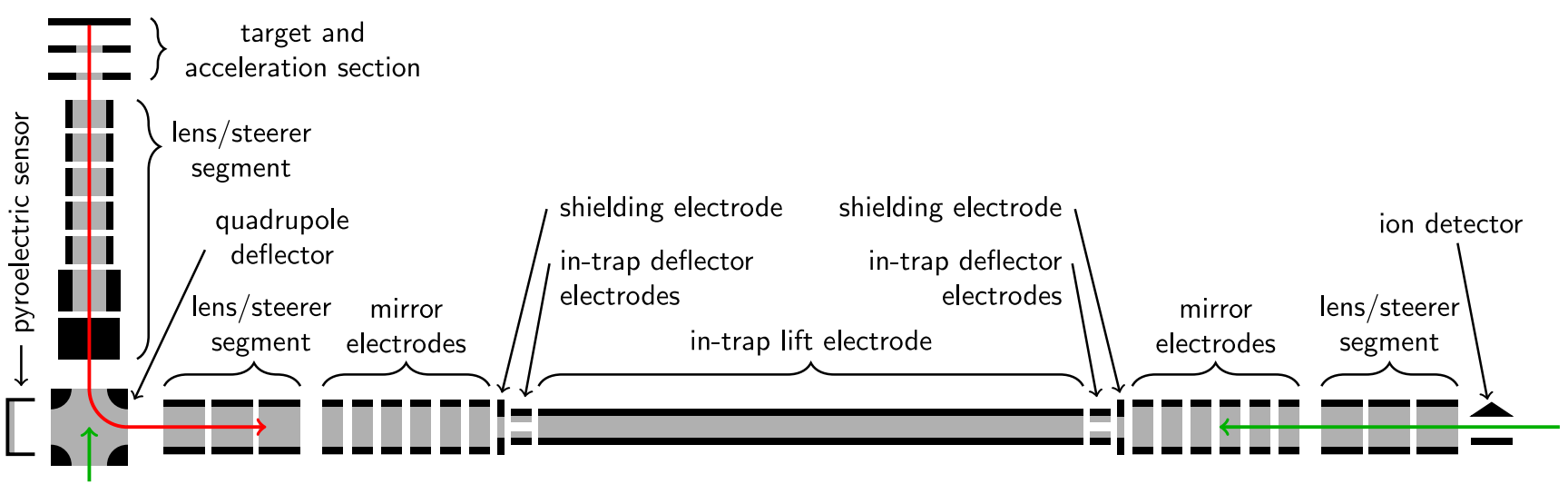

FIG. 1. Overview of the experimental setup, with ion path indicated in red, source and interaction laser beams in green.

this one naturally occurring isotope. Thus, the challenge of the fragment analysis never rises beyond that of cluster sizes $\mathrm{Bi}_{n}$, where $n$ denotes the number of atoms in the molecule. For small clusters, the required mass resolving power of the second MS stage is consequently only on the order of $R=m / \Delta m \lesssim 20$, which is easily accessible with linear ToF MS [18]: The ions were released by switching off the exitside mirror potential simultaneously to the excitation-laser pulse to record their "single-path" flight times to the detector. The overall experimental cycle can be described as MR-ToF MS/ToF MS. The high resolving power of the MR-ToF operation is needed in the first stage to suppress contaminant ions in the form of low-abundance compound clusters $\mathrm{Bi}_{n-m} \mathrm{~Pb}_{m}$ $(m<n)$ or, in the general case, molecular isotopes ("isotopologues") [38] and ensure unambiguous results. For the second step of product ion analysis, the low resolving power of linear ToF MS is fully sufficient.

For the present study of lead-doped bismuth clusters, the laser-ablation target is purposely changed to an alloy containing $25 \mathrm{at} \% \mathrm{~Pb}$ and 75 at $\% \mathrm{Bi}$ to enhance their production rate and select them for photoexcitation. Fragmentation of a compound cluster is inherently more complex and can be understood as the loss of a neutral (compound) cluster from the selected $n$-atomic precursor, i.e.,

$$
\mathrm{Bi}_{n-m} \mathrm{~Pb}_{m}^{+} \rightarrow \mathrm{Bi}_{n-m-x} \mathrm{~Pb}_{m-y}^{+}+\mathrm{Bi}_{x} \mathrm{~Pb}_{y},
$$

where $x, y \in\{0,1,2, \ldots\}$ and $x \leqslant n-m, y \leqslant m$. Note that it is impossible to investigate such behaviors in the previous MR-ToF MS/ToF MS-style measurement [31], as the mass resolving power needed to distinguish the charged product species quickly rises to $R>1000$ with increasing $n$.

While there are other approaches of tandem mass spectrometry, they offer no viable alternative for cases like the present. Quadrupole mass filters (QMFs), in particular "triple quad" setups [39], have been used to investigate metal clusters $[40,41]$. In addition, they have also been employed for precursor selection prior to, e.g., (reflectron-)ToF MS [42,43]. However, limitations imposed by QMF devices include a low mass resolving power on the order of a few hundred and a typical maximum $m / q$ ratio below $10000 \mathrm{u} / e$. Fourier-transform ion-cyclotron resonance (FT-ICR) mass spectrometers are another option for (among others) cluster studies [44-46], as they offer excellent resolving powers reaching up to several millions $[47,48]$. However, ICR measurements are based on high magnetic fields provided by superconducting magnets, which can complicate optical access to the interaction region. In addition, the magnetic fields lead to $m / q$ restrictions [47,49], typically on the order of a few thousand atomic mass units over elementary charges, and a limited sensitivity. Several tens or hundreds of ions are needed per experimental cycle unless the ions have exceedingly high charge states or the detection is restricted to only very narrow bandwidths.

MR-ToF devices, in contrast, perform single-ion counting with negligible background, allowing measurements even for species with very low count rates (in the present study, only one $\mathrm{Bi}_{5}^{+}$event is observed in about 3000 cycles; see Fig. 4 below) that are inaccessible to ICR spectrometers. At the same time, MR-ToF resolving powers can be pushed to several 100000 [50].

Here, we report the introduction of an advanced measurement scheme: After the high-resolution MR-ToF precursor selection and subsequent cluster-laser interaction, the ions are stored further for high-resolution mass analysis. For a demonstration of the (MR-ToF MS) ${ }^{2}$ scheme, ${ }^{209} \mathrm{Bi}_{7}{ }^{208} \mathrm{~Pb}^{+}$ with mass number $A=1671$ is selected as precursor to characterize the change in its fragmentation behavior with respect to the pure octamer, ${ }^{209} \mathrm{Bi}_{8}^{+}$with $A=1672$. The first MS stage accordingly calls for a separation resolving power $R \gtrsim 2000$, which is easily surpassed with the present value of $R \approx 10000$ at 400 revolution periods [38].

\section{RESULTS AND DISCUSSION}

Figure 2 shows fragmentation spectra for both species of interest measured without additional post-excitation storage. While the pure cluster (a) has already been discussed in detail [31], the resolving power is not sufficient for the compound (b): Namely, the unresolved fragment species with mass difference $\Delta m=1 \mathrm{u}$, marked in violet, yellow, and orange, prevent comprehensive evaluation of the dissociation pathways. While the relative change in fragment abundances between the precursor species is obvious (see also values listed in Table I), 


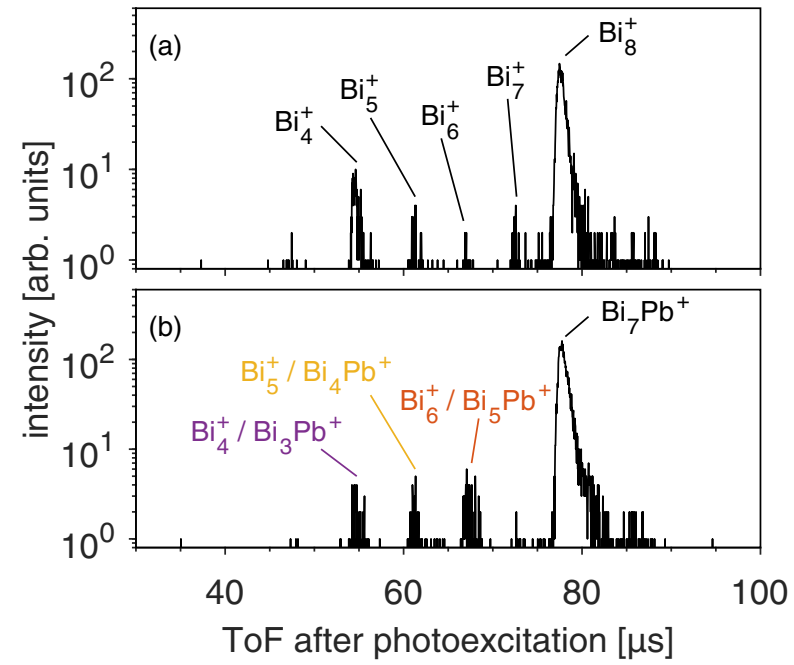

FIG. 2. Single-path time-of-flight spectra of $\mathrm{Bi}_{8}^{+}$(a) and $\mathrm{Bi}_{7} \mathrm{~Pb}^{+}$ (b) after photoexcitation at $\lambda=532 \mathrm{~nm}$ as introduced in Ref. [31]. Note that the resolving power is not sufficient to distinguish the $\mathrm{Bi}$ cluster fragments from the $\mathrm{Bi}-\mathrm{Pb}$ compounds in the bottom spectrum (marked violet, yellow, and orange).

the branching ratios

$$
B=\frac{I_{\mathrm{BiPb}}}{I_{\mathrm{BiPb}}+I_{\mathrm{Bi}}}
$$

for lead-containing fragments, where $I_{\mathrm{BiPb}}$ and $I_{\mathrm{Bi}}$ are the intensities of the compound and pure cluster ion species, respectively, are not accessible from the single-path spectrum.

In order to determine these ratios, the experimental cycle is changed to the (MR-ToF MS $)^{2}$ mode by delaying the ions' ejection after the photoexcitation for several tens to hundreds of additional revolution periods. All fragments are retained and lap each other according to their mass-to-charge ratio. The pure and compound species for the same fragment sizes separate at sufficiently high (post-excitation) revolution numbers, as illustrated in Fig. 3 for the $n=5$ species.

A single measurement has been performed with an additional post-excitation storage time corresponding to 162,145 , and 132 revolutions of the $n=4,5$, and 6 fragment species, respectively (Fig. 4). This storage time has been chosen such that all species of interest that appear in the spectrum are sufficiently resolved and do not overlap. The present mass resolving power in this second MS stage is $R \approx 10000$ and thus sufficient to distinguish all fragment species. This allows the determination of the branching ratios $B$ defined in Eq. (2). A separate measurement was performed for the low-

TABLE I. Relative fragment abundances in Fig. 2. The value for fragment cluster sizes $n$ includes the compound clusters $\mathrm{Bi}_{n-1} \mathrm{~Pb}^{+}$in the case of the $\mathrm{Bi}_{7} \mathrm{~Pb}^{+}$precursor. The abundances of $n \leqslant 3$ fragment ions are negligible.

\begin{tabular}{lcccc}
\hline \hline & $n=4$ & $n=5$ & $n=6$ & $n=7$ \\
\hline $\mathrm{Bi}_{8}^{+}$ & $0.63(3)$ & $0.16(2)$ & $0.06(1)$ & $0.12(2)$ \\
$\mathrm{Bi}_{7} \mathrm{~Pb}^{+}$ & $0.31(3)$ & $0.23(3)$ & $0.42(3)$ & $0.04(1)$ \\
\hline \hline
\end{tabular}

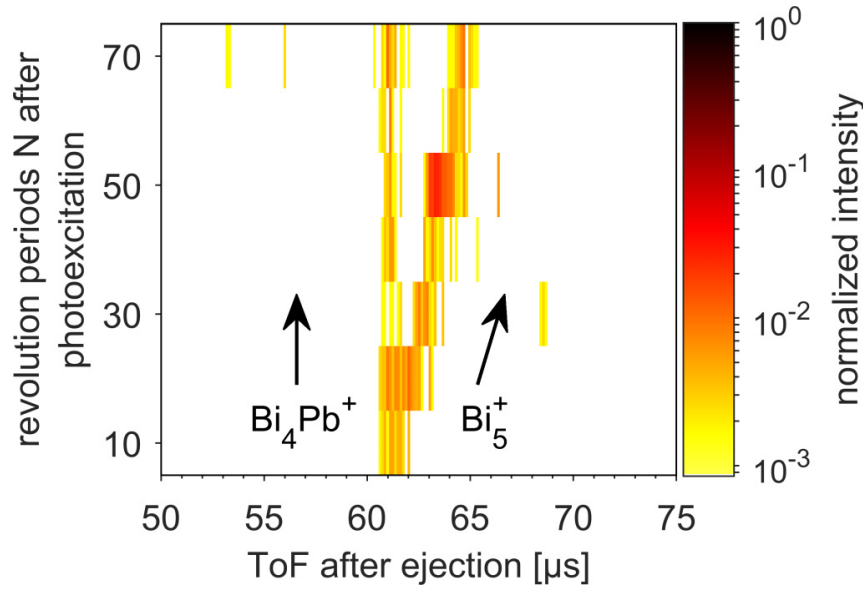

FIG. 3. Color-intensity $N$-versus-ToF plot [51,52] showing the separation of cluster species for $n=5$ fragments over several tens of post-excitation revolution periods.

abundance $n=7$ fragment at 84 post-excitation revolution periods. All values are listed in Table II and illustrated in Fig. 5.

For a purely statistical fragmentation process, i.e., with identical probabilities for all possible pathways, simple atom counting yields a branching ratio

$$
B_{\text {stat }}=\frac{n_{\text {frag }}}{n_{\text {frag }}+\left(n_{\text {prec }}-n_{\text {frag }}\right)}=\frac{n_{\text {frag }}}{n_{\text {prec }}}
$$

for lead-containing ions, where $n_{\text {frag }}$ and $n_{\text {prec }}$ are the sizes of the fragment and precursor cluster ions, respectively. Since $I_{\mathrm{BiPb}} \sim n_{\text {frag }}$ and $I_{\mathrm{Bi}} \sim\left(n_{\text {prec }}-n_{\text {frag }}\right)$, the above equation follows directly from (2). Comparing the experimental results (Table II) to $B_{\text {stat }}$, it is readily apparent that both the $n=4$ and $n=6$ fragment species exhibit a ratio skewed toward higher values, that is, toward more compound clusters than naively expected. Analogously, the $n=5$ and 7 species show lower

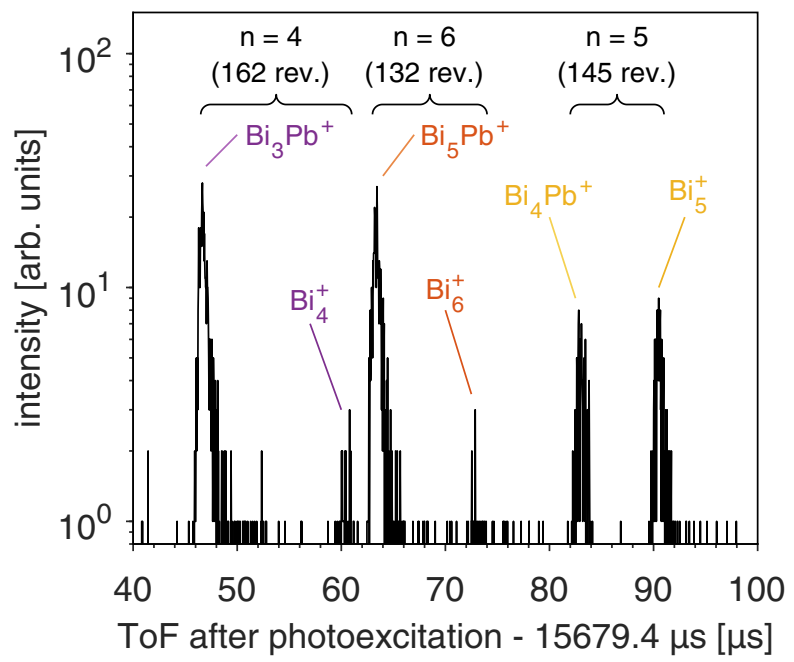

FIG. 4. Multi-reflection time-of-flight spectrum with $15679.4 \mu$ s of additional storage time after the photoexcitation of $\mathrm{Bi}_{7} \mathrm{~Pb}^{+}$. Signals with identical label colors are resolved species of the respective mixtures in Fig. 2. 
TABLE II. Measured branching ratios $B$ for fragment clusters resulting from the photoexcitation of $\mathrm{Bi}_{7} \mathrm{~Pb}^{+}$and expected ratios $B_{\text {stat }}$ for a purely statistical dissociation behavior.

\begin{tabular}{lllll}
\hline \hline & $n=4$ & $n=5$ & $n=6$ & $n=7$ \\
\hline$B$ & $0.94(1)$ & $0.44(3)$ & $0.95(1)$ & $0.46(6)$ \\
$B_{\text {stat }}$ & 0.500 & 0.625 & 0.750 & 0.875 \\
\hline \hline
\end{tabular}

values, correlating to a preference of the pure cluster retaining the charge.

Closed geometrical structures [53] or electronic shells [54] are known to yield clusters associated with "magic numbers," most prominently found by Knight et al. in sodium-cluster abundance spectra [55]. More importantly for the present case, a set of electron counting rules ("Wade's rules" [56]) is known to lead to increased stability if the number of a cluster's valence $p$ electrons equals $2 n+2$ (closo-), $2 n+4$ (nido-), or $2 n+6$ (arachno-species). $\mathrm{Bi}$ and $\mathrm{Pb}$ supply 3 and 2 of these "skeletal electrons" per atom due to their $s^{2} p^{2}$ and $s^{2} p^{3}$ configuration, respectively. Recently, density functional calculations have found that stable $\mathrm{Bi}-\mathrm{Pb}$ compound clusters are expected to follow these rules to a very high degree [57].

The deviation of the measured branching ratios $B$ from their element-abundance-based values $B_{\text {stat }}$ can be understood qualitatively in the framework of these studies. For the example of the $n=4$ fragments, the possible pathways with respect to the retention of the charge result in $\mathrm{Bi}_{3} \mathrm{~Pb}^{+}+\mathrm{Bi}_{4}$ or $\mathrm{Bi}_{3} \mathrm{~Pb}+\mathrm{Bi}_{4}^{+}$. Note that the charged compound cluster $\mathrm{Bi}_{3} \mathrm{~Pb}^{+}$has 10 valence $p$ electrons $(2 n+2)$ and the neutral bismuth tetramer $14(2 n+4)$. Both fragments are expected to show increased stability within the context of Wade's rules. In fact, neutral $\mathrm{Bi}_{4}$ (and $\mathrm{Bi}_{2}$ to a lesser extend) is known to be exceptionally stable from previous fragmentation studies [31,58-61], neutral-cluster vapors [62-64], and density functional calculations [65-68]. In contrast, the alternative pathway leads to two fragments with odd electron numbers, which are expected to be less stable. Thus, the high abundance of the charged compound cluster arises from favorable product configurations.

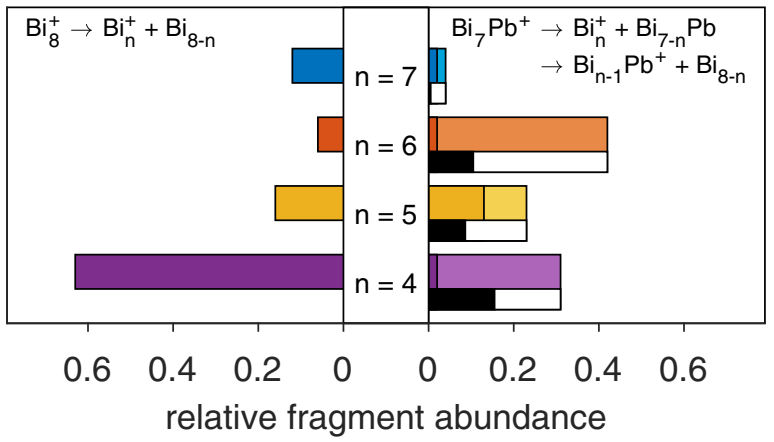

FIG. 5. Fragment abundances from photoexcitation of the pure (left) and compound (right) precursor species. For each fragment size $n$, i.e., total number of atoms in the fragment cluster, the pure species' abundance is given in darker, the compound's in lighter color. The black-and-white bars on the right indicate the purely statistical ratios according to $B_{\text {stat }}$ (see text).
Since the difference in the number of valence electrons between $\mathrm{Bi}$ and $\mathrm{Pb}$ is one, the above trend holds for all cluster sizes $n$ : One cluster species (pure or compound) will exhibit an even number of electrons while the other shows an odd number. The stable species alternates with $n$. Thus, the $n=6$ fragment branching ratio behaves analogously to that of $n=4$ while the pathway with the charge being retained on the pure cluster is favored for $n=5$ and 7. In the latter cases, this leads to lower branching ratios than calculated from pure statistics, in agreement with Table II.

Thus, the $B$ values are fully explained by the fragmentation behavior resulting from the substituted lead atom (Figs. 2 and 5). For the pure bismuth cluster, it is largely defined by the high stability of the neutral tetramer: $\mathrm{Bi}_{8}^{+} \rightarrow \mathrm{Bi}_{4}^{+}+\mathrm{Bi}_{4}$ has $63(3) \%$ fragment abundance. Following this pathway, the decays leading to stable configurations of the charged fragments $n=5[16(2) \%]$ and $n=7$ [12(2)\%] are preferred. The dissociation leading to the charged $n=6$ fragment with its odd number of valence electrons is least frequent [6(1)\%].

In contrast, due to the loss of a valence electron from the substitution, the compound cluster's most abundant pathways are the break-off of a neutral bismuth dimer and tetramer,

$$
\begin{aligned}
\mathbf{B i}_{7} \mathbf{P b}^{+} & \rightarrow \mathbf{B i}_{7}^{+}+\mathrm{Pb} 2(1) \% \\
& \rightarrow \mathrm{Bi}_{6} \mathrm{~Pb}^{+}+\mathrm{Bi} 2(1) \% \\
& \rightarrow \mathrm{Bi}_{6}^{+}+\mathrm{BiPb} 2(1) \% \\
& \rightarrow \mathbf{B i}_{5} \mathbf{P b}^{+}+\mathbf{B i}_{2} 40(3) \% \\
& \rightarrow \mathbf{B i}_{5}^{+}+\mathbf{B i}_{2} \mathbf{P b} 13(2) \% \\
& \rightarrow \mathrm{Bi}_{4} \mathrm{~Pb}^{+}+\mathrm{Bi}_{3} 10(1) \% \\
& \rightarrow \mathrm{Bi}_{4}^{+}+\mathrm{Bi}_{3} \mathrm{~Pb}_{2}(1) \% \\
& \rightarrow \mathbf{B i}_{3} \mathbf{P b}^{+}+\mathbf{B i}_{\mathbf{4}} 29(3) \%,
\end{aligned}
$$

as either leads to stable configurations for both the neutral and charged fragment. The clusters with increased stability are indicated in bold in the reaction equation (4), where the relative abundances of the corresponding fragment ions have been included. Compared to the pure cluster (see values in Table I), the loss of $\mathrm{Bi}_{4}$ is $34 \%$ less abundant while the loss of $\mathrm{Bi}_{2}$ is more abundant by the same amount. This likely results from the very high stability of the $\mathrm{Bi}_{5} \mathrm{~Pb}^{+}$ion: Across the investigated $(n, m)$ space for $\mathrm{Bi}_{n-m} \mathrm{~Pb}_{m}$, Seifried et al. have found only two clusters with a single lead atom fulfilling all imposed stability criteria (see Fig. 6 in Ref. [57]), $\mathrm{Bi}_{5} \mathrm{~Pb}^{+}$ and the present precursor $\mathrm{Bi}_{7} \mathrm{~Pb}^{+}$. The present measurement thus agrees with their findings based on density functional calculations. The next dominant fragmentation pathways of the compound cluster in terms of abundance are those yielding charged $n=5$ fragments, where both competing channels are about equally populated [10(1)\% and 13(2)\%]. All remaining pathways are negligible in terms of their abundances.

In particular, the abundance of the $n=7$ products decreases significantly compared to the analog photofragmentation of the pure $\mathrm{Bi}$ precursor, even though the more highly abundant $\mathrm{Bi}_{8}^{+} \rightarrow \mathrm{Bi}_{7}^{+}+\mathrm{Bi}$ pathway leads to an unfavorable odd-numbered neutral bismuth atom. However, by this neutral-atom emission $\mathrm{Bi}_{8}^{+}$turns into an even-numbered and thus more stable species. In contrast, $\mathrm{Bi}_{7} \mathrm{~Pb}^{+}$has been proposed to have a very high stability itself [57]. Thus, for this 
stable compound cluster, neutral-atom break-off is found to be less favorable than the competing decay channels by the present investigations.

\section{CONCLUSIONS}

In summary, the present studies have unveiled stability changes resulting from doping atomic bismuth clusters with a lead atom. The measurements were possible only due to the introduction of an advanced experimental technique, namely tandem multi-reflection time-of-flight mass spectrometry $(\mathrm{MR}-\mathrm{ToF} \mathrm{MS})^{2}$, which combines high-resolution precursor selection and high-resolution fragment analysis in a single device with very high sensitivity. This development opens the door to the study of reactions that have so far been inaccessible.
[1] R. Johnston, Atomic and Molecular Clusters (Taylor \& Francis, Abingdon, United Kingdom, 2002).

[2] J. Alonso, Structure and Properties of Atomic Nanoclusters, 2nd ed. (Imperial College Press, London, United Kingdom, 2011).

[3] M. M. Kappes, P. Radi, M. Schär, and E. Schumacher, Probes for electronic and geometrical shell structure effects in alkalimetal clusters: Photoionization measurements on $\mathrm{K}_{x} \mathrm{Li}, \mathrm{K}_{x} \mathrm{Mg}$, and $\mathrm{K}_{x} \operatorname{Zn}(x<25)$, Chem. Phys. Lett. 119, 11 (1985).

[4] S. Pollack, C. R. C. Wang, T. A. Dahlseid, and M. M. Kappes, Optical absorption probes of $\mathrm{Li}_{x} \mathrm{Na}_{4-x}(x=1,2)$ : Geometry, substitution isomers, and composition-dependent electron delocalization, J. Chem. Phys. 96, 4918 (1992).

[5] K. Hoshino, T. Naganuma, Y. Yamada, K. Watanabe, A. Nakajima, and K. Kaya, Ionization potentials of cobalt-sodium bimetallic clusters $\left(\mathrm{Co}_{n} \mathrm{Na}_{m}\right)$, J. Chem. Phys. 97, 3803 (1992).

[6] C. Yeretzian, Electronic structure effects in bimetallic $\mathrm{M}_{x} \mathrm{~N}$ clusters $(\mathrm{M}=$ alkali, $\mathrm{N}=$ divalent metal), J. Phys. Chem. 99, 123 (1995).

[7] U. Heiz, A. Vayloyan, E. Schumacher, C. Yeretzian, M. Stener, P. Gisdakis, and N. Rösch, $\mathrm{Na}_{x} \mathrm{Au}$ and $\mathrm{Cs}_{x} \mathrm{Au}$ bimetal clusters: Finite size analogs of sodium-gold and cesium-gold compounds, J. Chem. Phys. 105, 5574 (1996).

[8] E. Janssens, H. Tanaka, S. Neukermans, R. E. Silverans, and P. Lievens, Two-dimensional magic numbers in mass abundances of photofragmented bimetallic clusters, New J. Phys. 5, 46 (2003).

[9] R. Ferrando, J. Jellinek, and R. L. Johnston, Nanoalloys: From theory to applications of alloy clusters and nanoparticles, Chem. Rev. 108, 845 (2008).

[10] R. Johnston, Metal Nanoparticles and Nanoalloys, Vol. 3 (Elsevier, Amsterdam, Netherlands, 2012).

[11] F. Calvo, Nanoalloys: From Fundamentals to Emergent Applications (Elsevier, Amsterdam, Netherlands, 2013).

[12] J. M. Thomas, B. F. G. Johnson, R. Raja, G. Sankar, and P. A. Midgley, High-performance nanocatalysts for single-step hydrogenations, Acc. Chem. Res. 36, 20 (2003).

[13] J.-H. Liu, A.-Q. Wang, Y.-S. Chi, H.-P. Lin, and C.-Y. Mou, Synergistic effect in an Au-Ag alloy nanocatalyst: $\mathrm{CO}$ oxidation, J. Phys. Chem. B 109, 40 (2005).

[14] D. Wang, A. Villa, F. Porta, L. Prati, and D. Su, Bimetallic gold/palladium catalysts: Correlation between nanostructure and synergistic effects, J. Phys. Chem. C 112, 8617 (2008).

[15] H. T. Le, S. M. Lang, J. D. Haeck, P. Lievens, and E. Janssens, Carbon monoxide adsorption on neutral and cationic vanadium doped gold clusters, Phys. Chem. Chem. Phys. 14, 9350 (2012).

[16] Y. Cao, R. Jin, and C. A. Mirkin, DNA-modified core-shell Ag/Au nanoparticles, J. Am. Chem. Soc. 123, 7961 (2001).
[17] K. L. Busch, G. L. Glish, and S. A. Mcluckey, Mass Spectrometry/Mass Spectrometry: Techniques and Applications of Tandem Mass Spectrometry, 1st ed. (Wiley-VCH, Weinheim, Germany, 1988).

[18] R. Cotter, Time-of-Flight Mass Spectrometry: Instrumentation and Applications in Biological Research (American Chemical Society, Washington, D.C., United States, 1997).

[19] H. Wollnik and M. Przewloka, Time-of-flight mass spectrometers with multiply reflected ion trajectories, Int. J. Mass Spectrom. 96, 267 (1990).

[20] W. H. Benner, A gated electrostatic ion trap to repetitiously measure the charge and $m / z$ of large electrospray ions, Anal. Chem. 69, 4162 (1997).

[21] D. Zajfman, O. Heber, L. Vejby-Christensen, I. Ben-Itzhak, M. Rappaport, R. Fishman, and M. Dahan, Electrostatic bottle for long-time storage of fast ion beams, Phys. Rev. A 55, R1577 (1997).

[22] R. Wolf, D. Beck, K. Blaum, C. Böhm, C. Borgmann, M. Breitenfeldt, F. Herfurth, A. Herlert, M. Kowalska, S. Kreim, D. Lunney, S. Naimi, D. Neidherr, M. Rosenbusch, L. Schweikhard, J. Stanja, F. Wienholtz, and K. Zuber, On-line separation of short-lived nuclei by a multi-reflection time-offlight device, Nucl. Instrum. Methods Phys. Res., Sect. A 686, 82 (2012).

[23] R. N. Wolf, D. Beck, K. Blaum, C. Böhm, C. Borgmann, M. Breitenfeldt, N. Chamel, S. Goriely, F. Herfurth, M. Kowalska, S. Kreim, D. Lunney, V. Manea, E. Minaya Ramirez, S. Naimi, D. Neidherr, M. Rosenbusch, L. Schweikhard, J. Stanja, F. Wienholtz, and K. Zuber, Plumbing Neutron Stars to New Depths with the Binding Energy of the Exotic Nuclide ${ }^{82} \mathrm{Zn}$, Phys. Rev. Lett. 110, 041101 (2013).

[24] F. Wienholtz, D. Beck, K. Blaum, C. Borgmann, M. Breitenfeldt, R. B. Cakirli, S. George, F. Herfurth, J. D. Holt, M. Kowalska, S. Kreim, D. Lunney, V. Manea, J. Menendez, D. Neidherr, M. Rosenbusch, L. Schweikhard, A. Schwenk, J. Simonis, J. Stanja, R. N. Wolf, and K. Zuber, Masses of exotic calcium isotopes pin down nuclear forces, Nature (London) 498, 346 (2013).

[25] O. Aviv, B. Kafle, V. Chandrasekaran, O. Heber, M. L. Rappaport, H. Rubinstein, D. Schwalm, D. Strasser, Y. Toker, and D. Zajfman, Absolute photo-destruction and photofragmentation cross section measurements using an electrostatic ion beam trap, Rev. Sci. Instrum. 84, 053106 (2013).

[26] C. Breitenfeldt, K. Blaum, S. George, J. Göck, G. GuzmánRamírez, J. Karthein, T. Kolling, M. Lange, S. Menk, C. Meyer, J. Mohrbach, G. Niedner-Schatteburg, D. Schwalm, L. Schweikhard, and A. Wolf, Long-Term Monitoring of the 
Internal Energy Distribution of Isolated Cluster Systems, Phys. Rev. Lett. 120, 253001 (2018).

[27] S. Ring, H. B. Pedersen, O. Heber, M. L. Rappaport, P. D. Witte, K. G. Bhushan, N. Altstein, Y. Rudich, I. Sagi, and D. Zajfman, Fourier transform time-of-flight mass spectrometry in an electrostatic ion beam trap, Anal. Chem. 72, 4041 (2000).

[28] K. G. Bhushan, S. C. Gadkari, J. V. Yakhmi, and V. C. Sahni, Electrostatic ion trap and Fourier transform measurements for high-resolution mass spectrometry, Rev. Sci. Instrum. 78, 083302 (2007).

[29] T. Doussineau, C. Yu Bao, C. Clavier, X. Dagany, M. Kerleroux, R. Antoine, and P. Dugourd, Infrared multiphoton dissociation tandem charge detection-mass spectrometry of single megadalton electrosprayed ions, Rev. Sci. Instrum. 82, 084104 (2011).

[30] R. T. Hilger, P. J. Wyss, R. E. Santini, and S. A. McLuckey, Absorption mode Fourier transform electrostatic linear ion trap mass spectrometry, Anal. Chem. 85, 8075 (2013).

[31] P. Fischer and L. Schweikhard, Photofragmentation of $\mathrm{Bi}_{n}^{+/-}$ clusters $(n=2-19)$ in an electrostatic ion beam trap, Eur. Phys. J. D 73, 105 (2019).

[32] R. L. Hettich, Structural investigations of aluminum cluster ions, $\mathrm{Al}_{n}^{-}(n=3-50)$, J. Am. Chem. Soc. 111, 8582 (1989).

[33] A. Drescher, J. Kitching, J. E. Crawford, J. K. P. Lee, and G. Thekkadath, Studies of $\mathrm{Sb}$ and $\mathrm{Bi}$ cluster produced by laser desorption, Z. Phys. D 19, 203 (1991).

[34] H. S. Kim, T. D. Wood, A. G. Marshall, and J. Lee, Production of gold cluster ions by laser desorption/ionization Fouriertransform ion cyclotron resonance mass spectrometry, Chem. Phys. Lett. 224, 589 (1994).

[35] C. Stoermer, J. Friedrich, and M. M. Kappes, Observation of multiply charged cluster anions upon pulsed UV laser ablation of metal surfaces under high vacuum, Int. J. Mass Spectrom. 206, 63 (2001).

[36] R. N. Wolf, G. Marx, M. Rosenbusch, and L. Schweikhard, Static-mirror ion capture and time focusing for electrostatic ionbeam traps and multi-reflection time-of-flight mass analyzers by use of an in-trap potential lift, Int. J. Mass Spectrom. 313, 8 (2012).

[37] P. Fischer, G. Marx, and L. Schweikhard, Multiple ion capture and separation in an electrostatic storage device, Int. J. Mass Spectrom. 435, 305 (2019).

[38] P. Fischer, S. Knauer, G. Marx, and L. Schweikhard, In-depth study of in-trap high-resolution mass separation by transversal ion ejection from a multi-reflection time-of-flight device, Rev. Sci. Instrum. 89, 015114 (2018).

[39] R. A. Yost and C. G. Enke, Triple quadrupole mass spectrometry for direct mixture analysis and structure elucidation, Anal. Chem. 51, 1251 (1979).

[40] K. P. Kerns, B. C. Guo, H. T. Deng, and A. W. Castleman, Collision induced dissociation of titanium-carbon cluster cations, J. Chem. Phys. 101, 8529 (1994).

[41] K. R. Asmis, M. Brümmer, C. Kaposta, G. Santambrogio, G. von Helden, G. Meijer, K. Rademann, and L. Wöste, Massselected infrared photodissociation spectroscopy of $\mathrm{V}_{4} \mathrm{O}_{10}^{+}$, Phys. Chem. Chem. Phys. 4, 1101 (2002).

[42] A. V. Loboda, A. N. Krutchinsky, M. Bromirski, W. Ens, and K. G. Standing, A tandem quadrupole/time-of-flight mass spectrometer with a matrix-assisted laser desorption/ionization source: Design and performance, Rapid Commun. Mass Spectrom. 14, 1047 (2000).

[43] K. Hirsch, J. T. Lau, P. Klar, A. Langenberg, J. Probst, J. Rittmann, M. Vogel, V. Zamudio-Bayer, T. Möller, and B. von Issendorff, X-ray spectroscopy on size-selected clusters in an ion trap: From the molecular limit to bulk properties, J. Phys. B 42, 154029 (2009).

[44] J. L. Elkind, F. D. Weiss, J. M. Alford, R. T. Laaksonen, and R. E. Smalley, Fourier transform ion cyclotron resonance studies of $\mathrm{H}_{2}$ chemisorption on niobium cluster cations, J. Chem. Phys. 88, 5215 (1988).

[45] P. A. Limbach, L. Schweikhard, K. A. Cowen, M. T. McDermott, A. G. Marshall, and J. V. Coe, Observation of the doubly charged, gas-phase fullerene anions $\mathrm{C}_{60}^{2-}$ and $\mathrm{C}_{70}^{2-}$, J. Am. Chem. Soc. 113, 6795 (1991).

[46] S. Peredkov, A. Savci, S. Peters, M. Neeb, W. Eberhardt, H. Kampschulte, J. Meyer, M. Tombers, B. Hofferberth, F. Menges, and G. Niedner-Schatteburg, X-ray absorption spectroscopy of mass-selected transition metal clusters using a cyclotron ion trap: An experimental setup for measuring XMCD spectra of free clusters, J. Electron Spectrosc. 184, 113 (2011).

[47] A. G. Marshall, Fourier transform ion cyclotron resonance mass spectrometry, Acc. Chem. Res. 18, 316 (1985).

[48] M. V. Buchanan and R. L. Hettich, Fourier transform mass spectrometry of high-mass biomolecules, Anal. Chem. 65, 245A (1993).

[49] L. Schweikhard, J. Ziegler, H. Bopp, and K. Lützenkirchen, The trapping condition and a new instability of the ion motion in the ion cyclotron resonance trap, Int. J. Mass Spectrom. 141, 77 (1995).

[50] F. Wienholtz, D. Atanasov, S. Kreim, V. Manea, M. Rosenbusch, L. Schweikhard, A. Welker, and R. N. Wolf, Towards ultrahigh-resolution multi-reflection time-of-flight mass spectrometry at ISOLTRAP, Phys. Scr. T166, 014068 (2015).

[51] P. Schury, Y. Ito, M. Wada, and H. Wollnik, Wideband mass measurements with a multi-reflection time-offlight mass spectrograph, Int. J. Mass Spectrom. 359, 19 (2014).

[52] S. Knauer, P. Fischer, G. Marx, B. Schabinger, L. Schweikhard, and R. Wolf, Multi-reflection time-of-flight mass spectrometry with combined in-trap lift capture and mirror-switch ejection, Int. J. Mass Spectrom. 423, 46 (2017).

[53] T. Martin, Shells of atoms, Phys. Rep. 273, 199 (1996).

[54] W. A. de Heer, The physics of simple metal clusters: Experimental aspects and simple models, Rev. Mod. Phys. 65, 611 (1993).

[55] W. D. Knight, K. Clemenger, W. A. de Heer, W. A. Saunders, M. Y. Chou, and M. L. Cohen, Electronic Shell Structure and Abundances of Sodium Clusters, Phys. Rev. Lett. 52, 2141 (1984).

[56] K. Wade, Structural and bonding patterns in cluster chemistry, Adv. Inorg. Chem. Rad. 18, 1 (1976).

[57] C. Seifried, L. Longo, P. Pollak, and F. Weigend, The chemical space of $\mathrm{Pb}_{N-n} \mathrm{Bi}_{n}$ and $\left(\mathrm{Pb}_{N-n} \mathrm{Bi}_{n}\right)^{+}$: A systematic study for $N=3-13$, J. Chem. Phys. 146, 034304 (2017).

[58] M. E. Geusic, R. R. Freeman, and M. A. Duncan, Photofragmentation of antimony and bismuth cluster cations at $248 \mathrm{~nm}$, J. Chem. Phys. 88, 163 (1988). 
[59] M. M. Ross and S. W. McElvany, Production and fragmentation of antimony and bismuth cluster ions, J. Chem. Phys. 89, 4821 (1988).

[60] T. Bernhardt, B. Kaiser, and K. Rademann, Fragmentation of clusters induced by collision with a solid surface: Comparison of antimony and bismuth cluster ions, Z. Phys. D 40, 327 (1997).

[61] R. Kelting, A. Baldes, U. Schwarz, T. Rapps, D. Schooss, P. Weis, C. Neiss, F. Weigend, and M. M. Kappes, Structures of small bismuth cluster cations, J. Chem. Phys. 136, 154309 (2012).

[62] F. J. Kohl and K. D. Carlson, Dissociation energies of bismuth-antimony molecules, J. Am. Chem. Soc. 90, 4814 (1968).

[63] R. Wheeler, K. Laihing, W. Wilson, and M. Duncan, Semimetal clusters: Laser vaporization and photoionization of antimony and bismuth, Chem. Phys. Lett. 131, 8 (1986).
[64] M. E. Geusic, R. R. Freeman, and M. A. Duncan, Neutral and ionic clusters of antimony and bismuth: A comparison of magic numbers, J. Chem. Phys. 89, 223 (1988).

[65] H. K. Yuan, H. Chen, A. L. Kuang, Y. Miao, and Z. H. Xiong, Density-functional study of small neutral and cationic bismuth clusters $\mathrm{Bi}_{n}$ and $\mathrm{Bi}_{n}^{+}(n=2-24), \mathrm{J}$. Chem. Phys. 128, 094305 (2008).

[66] L. Gao, P. Li, H. Lu, S. F. Li, and Z. X. Guo, Size- and chargedependent geometric and electronic structures of $\mathrm{Bi}_{n}\left(\mathrm{Bi}_{n}^{-}\right)$ clusters $(n=2-13)$ by first-principles simulations, J. Chem. Phys. 128, 194304 (2008).

[67] J. M. Jia, G. B. Chen, D. N. Shi, and B. L. Wang, Structural and electronic properties of $\mathrm{Bi}_{n}(n=2-14)$ clusters from densityfunctional calculations, Eur. Phys. J. D 47, 359 (2008).

[68] D. Liang, W. Shen, C. Zhang, P. Lu, and S. Wang, Structural, electronic, vibrational and optical properties of $\mathrm{Bi}_{n}$ clusters, Mod. Phys. Lett. B 31, 1750260 (2017). 